\title{
Biomimetic Cellulosomes Assembled on Molecular Brush Scaffolds: Random Complexes vs. Enzyme
}

\section{Mixtures}

Oksana Zholobko ${ }^{1,6}$, Ademola Hammed ${ }^{2}$, Andrey Zakharchenko ${ }^{3}$, Nikolay Borodinov ${ }^{4}$ Igor Luzinov, ${ }^{4 *}$ Breeanna Urbanowicz ${ }^{5 *}$, Taras Patsahan ${ }^{7}$, Jaroslav Ilnytskyi ${ }^{8}$, Sergiy Minko,${ }^{3 *}$ Scott W. Pryor ${ }^{2 *}$, Andriy Voronov ${ }^{1 *}$

${ }^{1}$ Coatings and Polymeric Materials Department, North Dakota State University, Fargo, ND, USA

${ }^{2}$ College of Engineering and Department of Agricultural and Biosystems Engineering, North Dakota State University, Fargo, ND, USA ${ }^{3}$ Nanostructured Materials Lab, University of Georgia, Athens, GA, USA

${ }^{4}$ Department of Materials Science, Clemson University, Clemson, SC, USA

${ }^{5}$ Complex Carbohydrate Research Center, University of Georgia, Athens, GA, USA

${ }^{6}$ Department of Mechanical Engineering, North Dakota State University, Fargo, ND, USA

${ }^{7}$ Department of Soft Matter Theory, Institute for Condensed Matter Physics of the National Academy of Sciences of Ukraine, Lviv, 79011, Ukraine

${ }^{8}$ Department of Computer Simulations of Many-Particle Systems, Institute for Condensed MatterPhysics of the National Academy of Sciences of Ukraine, Lviv, 79011, Ukraine

\section{Corresponding Authors}

*Breeanna Urbanowicz, Complex Carbohydrate Research Center, University of Georgia, Athens, GA; orcid.org/ 0000-0001-5247-4513; Email: breeanna@uga.edu

*Igor Luzinov, Department of Materials Science, Clemson University, Clemson, SC, USA; orcid.org/ 0000-0002-1604-6519; Email: luzinov@clemson.edu

*Sergiy Minko, Nanostructured Materials Lab, University of Georgia, Athens, GA; orcid.org/ 0000-0002-7747-9668; Email: sminko@uga.edu 
*Scott W. Pryor, Agricultural and Biosystems Engineering, North Dakota State University, Fargo, ND; Email: scott.pryor@ndsu.edu

*Andriy Voronov, Coatings and Polymeric Materials Department, North Dakota State University, Fargo, ND; E-mail: andriy.voronov@ndsu.edu

Number of figures 5

Number of tables 1 
NMR spectroscopy of the MB copolymers. Copolymer samples for ${ }^{1} \mathrm{H}$ NMR spectroscopy were prepared by dissolving in deuterated water. ${ }^{1} \mathrm{H}$ NMR spectra were recorded with an AVANCE III HDTM $400 \mathrm{MHz}$ high-performance digital NMR spectrometer at $22.5^{\circ} \mathrm{C}$. The spectra were referenced to a TMS signal as an internal standard.

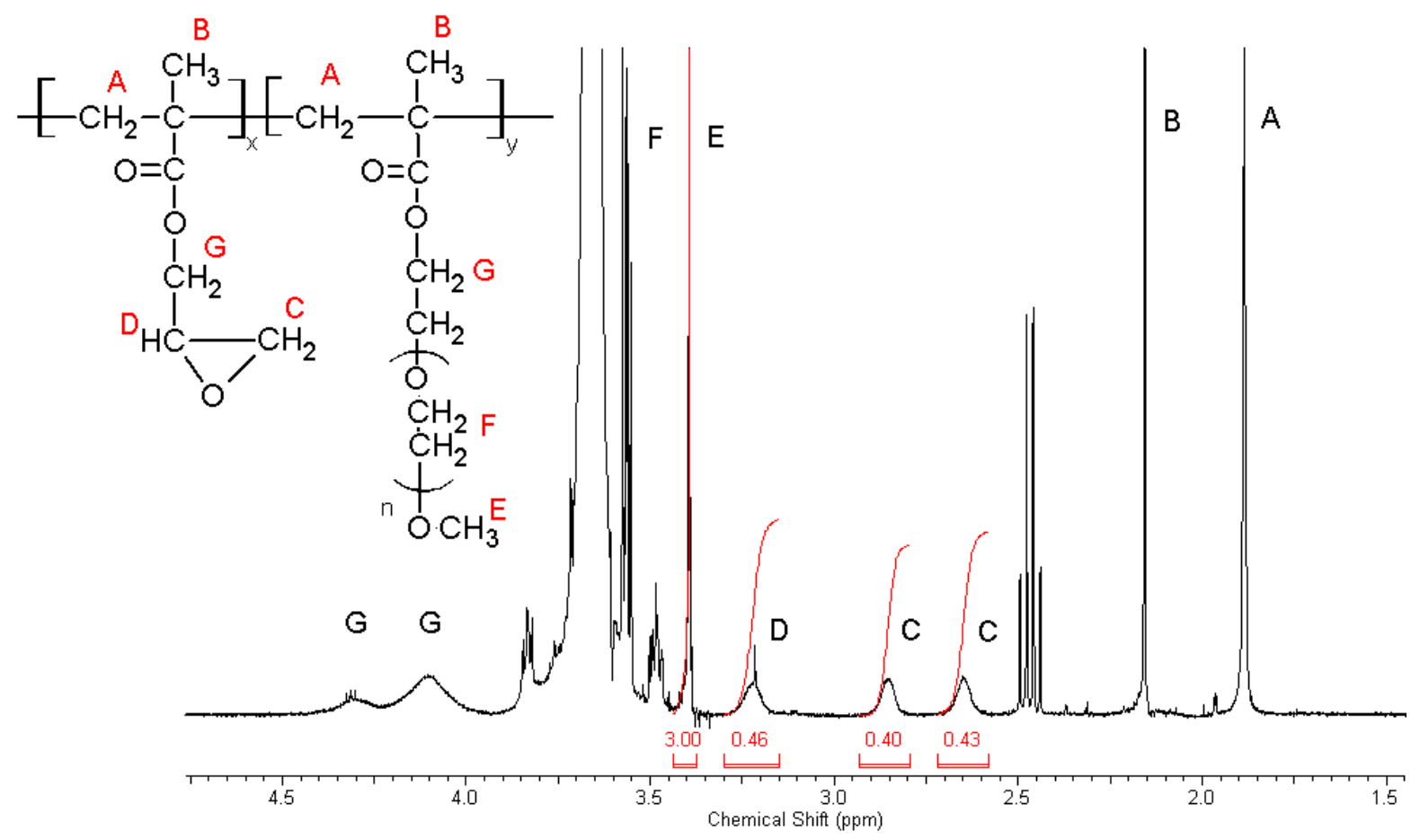

Figure S1. ${ }^{1} \mathrm{H}$ NMR spectrum of poly(GMA-co-PEGMA).

Table S1. Conjugation efficiency for $\mathrm{GP}_{300}$ EPC for different polymer:enzyme ratios

\begin{tabular}{c|cccccccc}
$\begin{array}{c}\text { GP } P_{300} \text { 0.3:0.7:Enzyme } \\
\text { ratio }\end{array}$ & $1: 1$ & $2.5: 1$ & $5: 1$ & $7.5: 1$ & $10: 1$ & $20: 1$ & $\mathbf{3 0 : 1}$ & $50: 1$ \\
\hline $\begin{array}{c}\text { Amount of conjugated } \\
\text { enzyme, \% }\end{array}$ & 59.1 & 66.5 & 86.4 & 82.4 & 82.9 & 88.6 & 86.2 & 89.1 \\
\hline
\end{tabular}




\section{MD-simulations}

We used the simulation technique termed as coarse-grained molecular dynamics, where the relevant groups of atoms are mimicked via soft spherical beads. The bead may represent a small fragment of a polymer chain, a larger entity such as a macromolecule (e.g. enzyme) or colloid particle, or a collection of solvent beads. The benefit is two-fold: (i) reduction of the degrees of freedom in a system, and (ii) soft nature of effective inter-bead potentials allowing longer timesteps of simulation. Both, being combined, allow covering larger length and time scales using moderate computing power expenditure as compared to the atom-based molecular dynamics. These benefits, however, came at the cost of losing the details on the atomistic level, literally, those that occur in each bead interior.

In this study we focus on a spatial arrangement of enzymes rather than on their internal structure and explicit functionality in hydrolysis. This emphasis allows us to treat all of them, of the types A, B, C and D, as soft spheres of the same diameter $\sigma_{e}$. The latter serves as the length scale of the simulated system, hence we set $\sigma_{e}=1$. Each enzyme contains a reactive site inside it, in the form of a small sphere of diameter $\sigma_{d}=0.27$. Molecular scaffolds are fully flexible polymers of the sidechain architecture with the functionality of 4 and single-bead side chains. Each side-chain bead contains a reactive site inside it, in the form of a small sphere of the same diameter as a matching reactive site of the enzyme, $\sigma_{c}=\sigma_{d}=0.27$. Solvent beads represent a collection of solvent molecules when the choice of their diameter is arbitrary. Our choice of $\sigma_{d}=1$ was dictated by the need to save on computer time, but other choices, e.g. $\sigma_{d}=0.5$, were also tried and found to be equally viable.

Two types of the interaction potentials between the beads, the soft repulsive, $V(r)$, and soft attractive, $W(r)$, were used, with their respective analytic forms given below:

$$
\begin{aligned}
& V(r)= \begin{cases}U\left(1-r^{*}\right)^{2}, & r^{*}<1 \\
0, & r^{*}>1\end{cases} \\
& W(r)=\left\{\begin{array}{lc}
U\left[\left(1-r^{*}\right)^{2}-\varepsilon^{*}\right], & r^{*}<1 \\
U\left[\left(1-r^{*}\right)^{2}-\varepsilon^{*}-\frac{(1-1 / q)^{q-1}}{q\left(\varepsilon^{*}\right)^{q-1}}\left|1-r^{*}\right|^{2 q}\right], & 1<r^{*}<r_{c}^{*} \\
0, & r^{*}>r_{c}^{*}
\end{array}\right.
\end{aligned}
$$

Here $r^{*}=\left(\sigma_{\mathrm{i}}+\sigma_{\mathrm{j}}\right) / 2$ is the reduced separation between the interacting $i$ th and $j$ th spheres with their respective diameters $\sigma_{\mathrm{i}}$ and $\sigma_{\mathrm{j}}, U=1$ provides the energy scale of the simulations, $\varepsilon^{*}$ and $r_{c}^{*}$ are the 
reduced well depth and a cutoff distance of the attractive interaction potential, respectively, whereas $q$ provides the range of its attractive tail. The shape of the $W(\mathrm{r})$ potential at various $q$ is shown in Fig. S2.

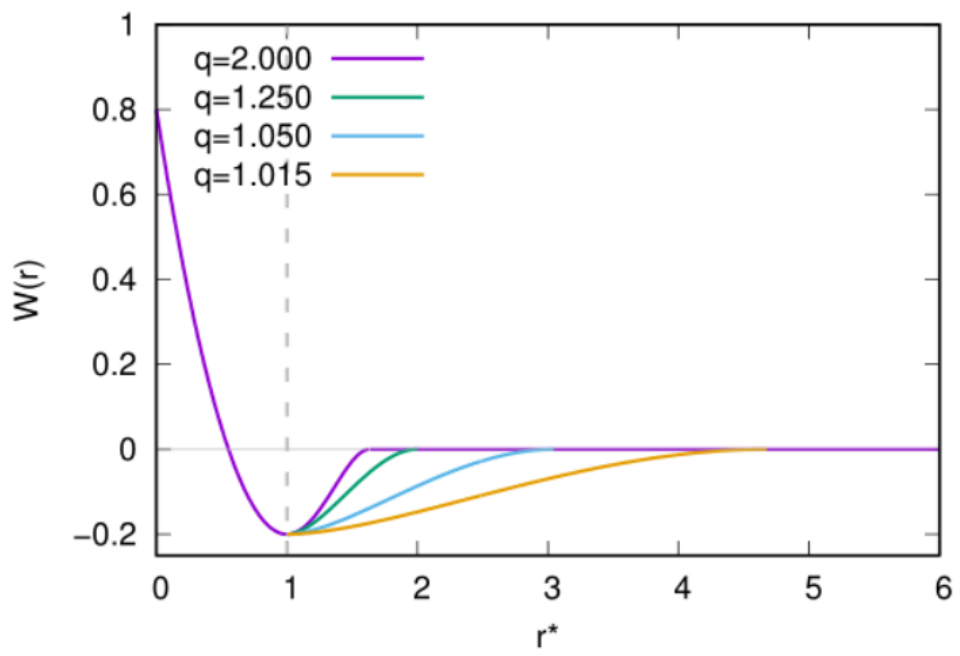

Figure S2. The shape of the attractive potential $W(\mathrm{r})$ shown at $U=1, \varepsilon^{*}=0.2$ (i.e. enzyme-polymer attractive strength is $\left.\varepsilon_{e p} / k_{B} T \sim 26\right)$ and at a range of values for $q$.

The simulations were performed in the NVT-ensemble in the simulation box with reduced dimensions of $L_{x}=L_{y}=L_{z}=18$ and two soft walls normal to the Z-axis and the periodic boundary conditions applied in the other two directions. Technically, each soft wall is introduced via the beads of the diameter $\sigma_{e}$ placed outside the simulation box next to the respective edge. Reduced temperature is $k_{B} T / U=0.0078$, and reduced timescale of the simulations is set as $\Delta t^{*}=7.5 \times 10^{-3}$, where the reduced time is defined as $t^{*}=t\left(m_{e}\left(\sigma_{e}\right)^{2} / U\right)^{1 / 2}$ and $m_{e}$ is the enzyme mass.

For the Mix simulations case (no polymer chains are present), all the pairwise bead-bead interactions and the bead-wall ones are soft repulsive, given by the $V(r)$ potential, except for the enzyme-wall interactions. The latter are described by the attractive $W(r)$ form with the well depth of $\varepsilon^{*}=0.07$ (i.e., enzyme-wall attractive strength is $\varepsilon_{e w} / k_{B} T \sim 9$ ). As a result, the enzymes gradually adsorb on both walls forming random instant motives, as shown in the snapshot in Fig. S3. The motifs are identified according to the spatial proximity of adjacent enzymes (e.g. the separation between them is less than $2 \sigma_{e}$ ). 


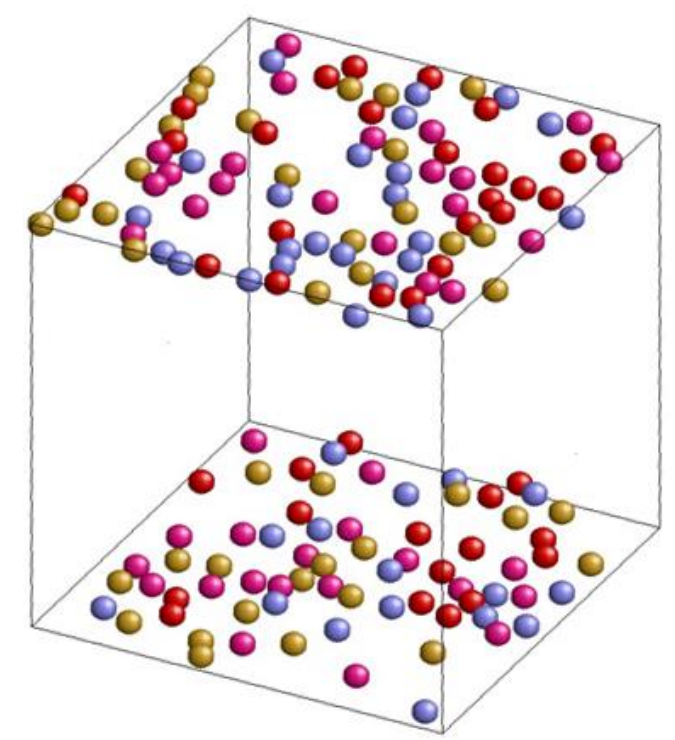

Figure S3. Snapshot of the Mix simulation showing enzymes adsorbed on both walls and formation of instant motifs of them (types A, B, C and D are shown via different colors). Solvent beads are not shown for the sake of clarity.

For the EPC simulations, the additional attractive interaction between the reactive beads of a polymer scaffold and these of enzymes are brought into play. These pairs interact by the same $W(r)$ potential but with the deeper well of $\varepsilon^{*}=0.2$ (i.e. enzyme-polymer attractive strength is $\varepsilon_{e p} / k_{B} T \sim 26$ ). This case mimics strong (either covalent of based on hydrogen bonding) attachment of enzymes to the scaffold chains and ensures that they do not detach due to the competing attraction to the walls. The reactive bead dimension, its placement within the host bead, and the set of respective interaction potentials between all participating beads are tuned to suppress the possibility to form more than one bond per one reactive bead.

The first stage of the EPC simulations (self-assembly of complexes) is illustrated in Fig. S4. Fig. S4a shows the initial stage in the form of a mixture between the enzymes and polymer scaffolds, whereas Fig. S4b displays the self-assembled complexes.

The second stage of the EPC simulations is shown in Fig. S5, illustrating adsorption of EPC on both walls and the motifs of enzymes, that are further identified following the same criteria as for the Mix simulations. 
(a)

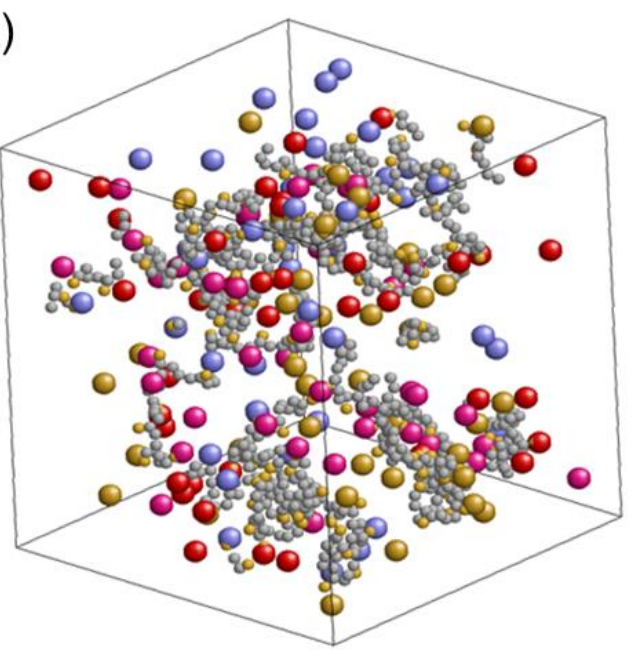

(b)

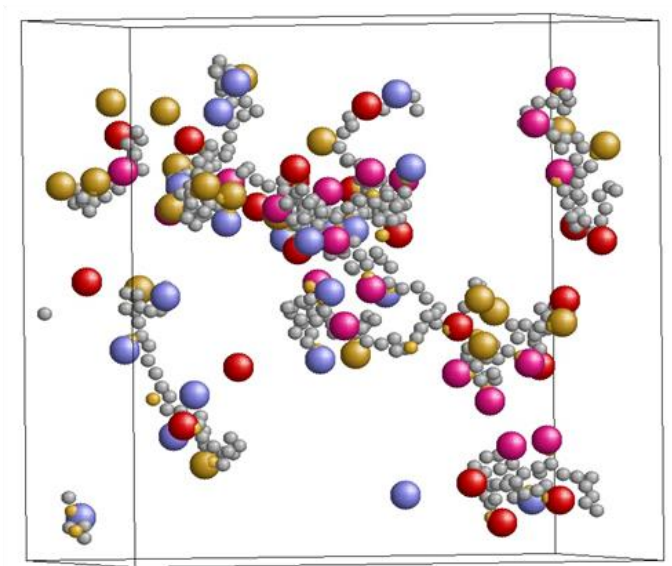

Figure S4. Snapshots of the first stage of the EPC simulation: (a) the initial stage of the enzymepolymer mixture ( $N_{e}=160$ case is illustrated), (b) the final stage with enzymes being complexed with polymer chains ( $N_{e}=80$ case is illustrated to avoid cluttering). Solvent beads are not shown for the sake of clarity.

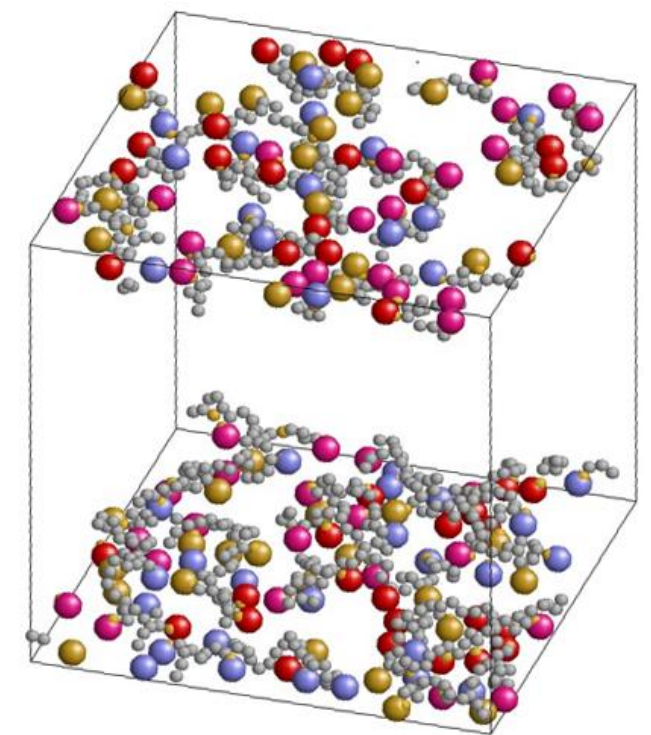

Figure S5. A snapshot of the second stage of the EPC simulation for a $N_{e}=160$ case. Adsorbed EPCs show patterns with various enzyme motifs formed due to their attachment order on the polymer scaffold and proximity between the complexes. Solvent beads are not shown for the sake of clarity. 